\title{
The effects of new 2030 scenario: reduction of short- circuit power and widening of voltage dips
}

\author{
L. Michi, E.M. Carlini, L. Caciolli, \\ D. Polinelli, P. Capurso, A. Proietti \\ Grid Development Department \\ Terna SpA \\ Roma, Italy \\ alessandro.proietti@terna.it
}

\author{
A. Berizzi, C. Bovo \\ Dipartimento di Energia \\ Politecnico di Milano \\ Milano (Italy) \\ alberto.berizzi@polimi.it
}

\begin{abstract}
In Italy, $80 \%$ of PV installations are at MV and LV levels, which makes it particularly challenging to control them from the national dispatch centre; this leads to an increase of the reverse power flow in the primary and secondary substations, increasing pressure on the existing measuring and protection systems and on voltage control. The National Strategic Plan, approved on November $10^{\text {th }}, 2017$ by the Ministry of Economic Development and the Ministry of the Environment, has launched an ambitious challenge, e.g., phasing-out of coal and the increase of electricity from renewable sources: more than $55 \%$ by 2030 of gross final consumption. The power system must be able to withstand unplanned events and manage their impacts on the network, thus reducing the risk of cascading effects and maintaining suitable quality of supply. During operation with a high percentage of RES connected to the network through inverters, the system is weaker (lower short circuit power) and, with currently adopted controls, less able to react to emerging faults. With the increase of PV installations, also due to reduction of rotating synchronous machines connected to transmission grid, there is lower Short-circuit-Power available and therefore voltage dips generated at transmission level have larger impact (c.g. area impacting supply quality widens hundreds of kilometers away from the event). This paper summarizes the main challenges in terms of impacting supply quality for the Italian Power system in a new scenario with more than $50 \%$ RES by 2030 of gross final consumption and suggests some computation procedures to investigate the phenomenon.
\end{abstract}

Index Terms Transmission and dispatching, system planning, RES integration, long term scenario, reverse power flow (RPF), fault voltage (FV) level, voltage control .

\section{INTRODUCTION}

In general, an increasing number of small-scale electricity generation connected to medium voltage (MV) and low voltage (LV) networks puts a large burden on the transmission and distribution grid systems which were originally designed for unidirectional, top-down, power flows.

In Italy, $80 \%$ of the PV (Photovoltaic) installations are at MV and LV levels, which makes it particularly challenging to control them from the national dispatch centre, and this leads to an increase of the Reverse Power Flow (RPF) in the primary and secondary substations, increasing pressure on the existing measuring and protection systems and on voltage control (Figure 1).

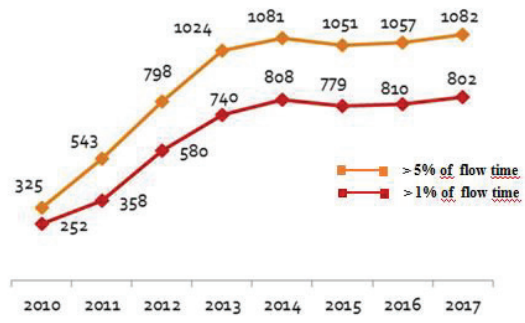

Figure 1 - Number of substation affected by reverse power flow for different reverse flow time "RPT" in the Italian distribution system

The grid must be able to withstand unplanned events and to manage their impacts on the network, thus reducing the risk of cascading effects and preserving quality of supply.

During operation with a high percentage of RES (Renewable Energy Systems), mostly connected to the network through inverters, the system is weaker (lower short circuit power) and less able to react to emerging faults unless special control logics are adopted. Due to the reduction of rotating machines connected to transmission grid, the short-circuit power available is lower and therefore voltage dips generated at transmission level have larger impact (in this simulation, the spatial distribution of DG - Distributed generation - has been assumed homogenous).

Among the main consequences of RES, in particular if installed at MV levels, in some points are highlighted: insensitivity of the grid, linked to the short-circuit power levels of the grid, which is foreseen to decrease as RES increase, thus resulting in a transmission grid more vulnerable to the faults. Flexibility, and, reduced inertia levels, that make the power system more vulnerable against ramps of PV in feed and frequency transients. In this paper, in particular, the analysis of the short-circuit power is considered. 


\section{SHORT-CIRCUIT-POWER EVOLUTION}

Currently Terna performs the short-circuit analyses according the Annex A8 [1] of Terna's Grid Code [2] which is based on standard CEI EN 60909-0. Generally, in the above traditional short-circuit method, the short circuit power and the profile of the voltages in the nodes of the grid during the faults depend mainly on the topology of the grid, the impedance of each grid branch, place and size of rotating machines connected to the grid.

In this kind of method, shunt branches contribution could also be taken into account.

Looking at the Italian scenario:

- In 2008 the RES penetration was low, with additional Wind installed capacity about $3.500 \mathrm{MW}$ and PV installed capacity less than $450 \mathrm{MW}$; rotating machines connected to the grid ensured high short-circuit power.

- In 2017, installed capacity was about 9.737 MW for wind and 19.682 MW for PV; meanwhile thermal power plants reduced more than $12 \mathrm{GW}$ in the last 5 years.

- In 2030, capacity is expected to reach about 16.200 MW and 46.600 MW respectively from wind and PV.

Due to the RES penetration increasing, the short-circuit power is generally supposed to decrease at most electric nodes and the short circuit-effect to impact on a wider area. To understand the impact of the new generation mix, the development of the grid is not included in the 2030 model. The impact of the short-circuit could be seen from two special points of view: the intensity and the extension of the phenomenon. In Sardinia, a 3-phase short-circuit has been simulated at $220 \mathrm{kV}$ of Villasor: it is possible to observe a general reduction of the fault voltage level (FV) in the 220$400 \mathrm{kV}$ grid, that can reduce more than $20 \%$ in 2030 compared to 2008 (Figure 2).

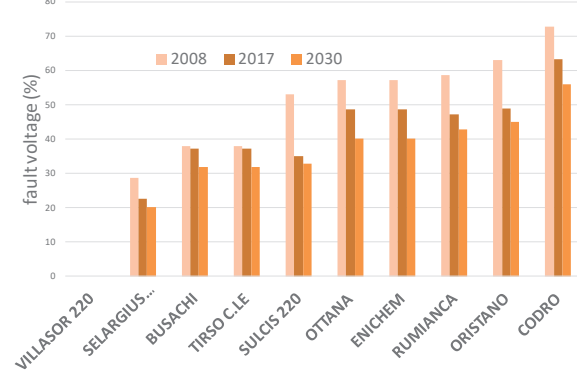

Figure 2 - Comparison of Fault Voltage ( $\%$ on the nominal voltage) with 3 -phase short circuit at $220 \mathrm{kV}$ Villasor busbar.

Another test was performed in the south of the Italian peninsula (Galatina $400 \mathrm{kV}$ substation): in Figure 4, the more and more important intensity of phenomena (decreased voltage level) in the closer substations between 2008 and 2030 is shown.

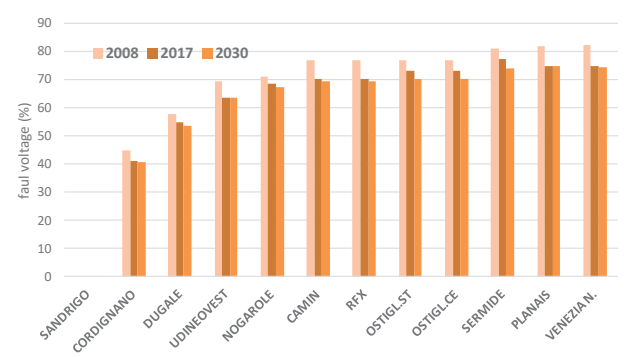

Figure 3 - Comparison of Fault Voltage ( $\%$ on the nominal voltage) with 3 -phase short circuit at $400 \mathrm{kV}$ Galatina busbar.
The characteristics of the grid and the evolution of the electric system in the north of Italy are different from the south and two main islands; this is evident in Figure 4, where it is clear that Sandrigo $400 \mathrm{kV}$ bus-bar behaviour is much less affected by the reducing of short-circuit power. This portion of the grid is quite well interconnected to the neighbouring country and more meshed.

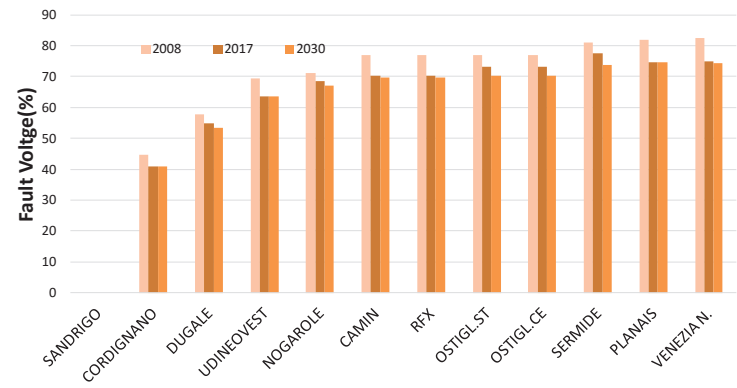

Figure 4 - Comparison of Fault Voltage ( $\%$ on the nominal voltage) with 3-phase short circuit at $400 \mathrm{kV}$ Sandrigo.

In Figure 5, a comparison was carried out, highlighting the areas in which the voltages reduces more than 60 (Sardinia) and $80 \%$ (North East and South) of nominal voltage.

The grey circles represents the impact of 2008 model, while the yellow ones represent the impact of a short circuit in a high RES penetration scenario such as 2030 time horizon.

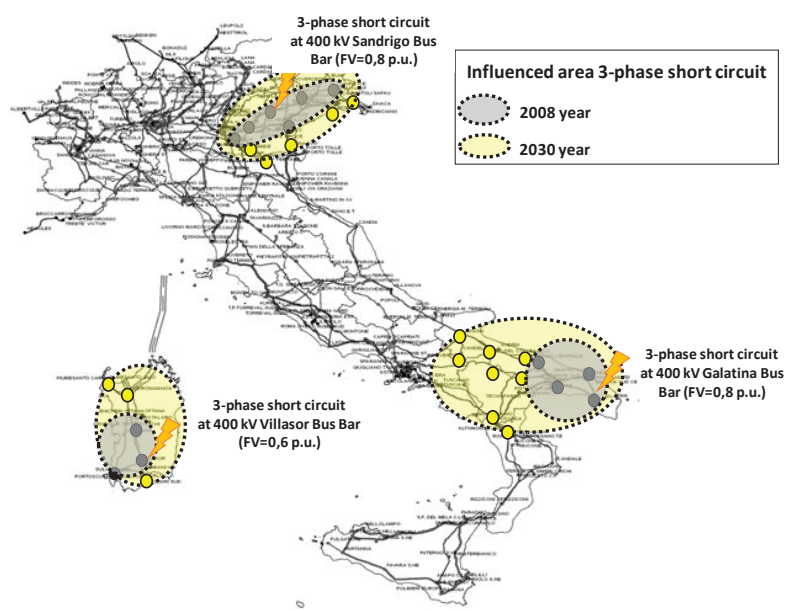

Figure 5 - Spatial impact of short circuit where voltage decrease up to $80 \%$.

\section{A PROPOSAL FOR A SHORT-CIRCUIT CURRENT} METHODOLOGY INCLUDING INVERTER-BASED GENERATION

The computation of short-circuit currents is typically made according to the Thévenin equivalent model of the power system, assuming all rotating machines contributing to shortcircuits depending on their subtransient reactances. Due to the importance of converter-based RES generation, this approach might be no longer valid. In order to get correct answers about short-circuit levels, dynamic simulation, including details of controls, should be used; however, in order to keep the traditional, simple and well-known steadystate method, a modified approach is proposed in the present paper. The importance of this approach is also highlighted in [3], where particular attention is devoted to steady-state modelling of RES for both power flow calculations and short-circuit studies. RES models to properly represent inverter-based contribution to short-circuit currents are needed, based on a current-limited Norton equivalent with 
control loops to model the response under abnormal voltage conditions. According to [3], traditional steady-state shortcircuit analysis is no longer adequate for inverter-based RES, and new iterative algorithms are needed.

\section{A. Full-scale converter}

Modern Wind Power Plants (WPP) are based on the fullscale converter (FSC). Their behaviour, in the presence of a short-circuit, is better represented by a current source rather than a constant voltage behind a reactance. If the residual voltage is high enough, the converter keeps operating according to the set control strategy, e.g., regulating real and reactive current components. On the contrary, if the voltage dip is more severe, after an initial transient overcurrent, the control limits the current within 1 or 2 cycles to a peak value within 2.0 and 4 p.u, Figure 6) and, after first 1 or 2 cycles, to a rms value within 1.1 and 1.3 p.u. This causes a transient imbalance between the power delivered to the grid and the mechanical power of the WT (Wind Turbine). This energy might result either in an acceleration of the shaft or in a dclink voltage increase. Moreover, It should be noted that the currents supplied by the FSCs tend to be in quadrature with the currents supplied by the rotating generators; therefore they have a modest influence on the value of the total shortcircuit current.

Given the dependence on the manufacturer's controls, in general, TSOs do not have means to compute exactly the contribution to short-circuit currents, the only information available being included in some charts depicting the maximum and minimum current magnitudes for different voltage levels and at different times after the fault.

\section{B. Doubly fed induction generator}

The behaviour of a Doubly Fed Induction Generator (DFIG) during short-circuit also depends on the residual voltage at the machine terminals [4][5][6]. Two contributions are important: the response of the IG to the voltage drop, comprising the current transient, and the converter control response, providing independent control of $\mathrm{P}$ and $\mathrm{Q}$ for the grid code compliance. If the voltage dip is small, the current transient is negligible, so that the DFIG is well represented by the converter control response. If this is unknown, it is possible to estimate it by the charts of the maximum and minimum current magnitudes, losing the information on the current phase angle. On the contrary, if the voltage drop is deeper ( 0.4 to 0.2 p.u.), the dc-link voltage becomes higher and higher, requiring additional measures to keep safe voltages, usually by means of a crowbar connected to the rotor terminals. Other solutions make use of a chopper resistance to the dc link. In both cases, the DFIG rotor controllability is lost and the generator behaves as a Wound Rotor IG, so that it can be modelled by a crowbar resistance in series to the transient reactance.

\section{Photovoltaic}

Being photovoltaic power plants connected to the grid through inverters, the short-circuit behaviour may be considered similar to the FSC-WTs. Particularly after an initial transient peak current up to 3 to $5 \mathrm{I}_{\mathrm{n}}$, the PV output is limited to a steady state (standard) value, typically $1.2 \div$ $1,4 I_{n}$ in 1-2 cycles, with negligible influence on the shortcircuit current, like for FSC.

\section{Wind Farms}

Usually, several WTs are connected together into Wind Farms (WF), so that an equivalent of the whole plant is required for system-wide short-circuit calculations. For FSC or DFIG, the grouping procedure is questionable. Under the assumptions that all turbines are of the same type, with similar parameters, operating under the same wind conditions, etc., it is acceptable to consider all power outputs to be in phase and, therefore, to just sum add them up. Otherwise, it is necessary to know the actual topology and the wind distribution to evaluate the contribution of every turbine and eventually the contribution at the Point of Common Coupling (PCC) and build an equivalent WF lumped model. This in general approach can be applied based on Grid Code requirements.

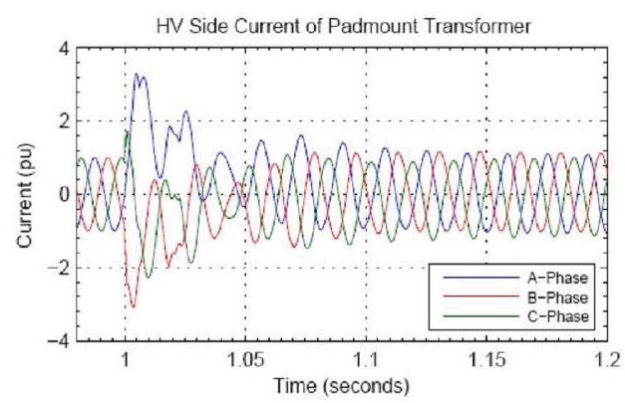

Figure 6 - Typical short-circuit current in a FSC.

\section{E. Grid Codes}

TSOs all over the world have recently modified their grid codes, requiring to RES both continuous operation even if the voltage dip reaches very low levels, and support to the voltage recovery by injecting reactive current. The typical requirements can be summarized in terms of Low Voltage Ride Through (LVRT) characteristics, restoration of real power with limited ramp after fault clearing, reactive current injection (RCI) for voltage support during fault and recovery.

This section presents an overview of the recent grid codes prescriptions, (mainly the Italian, German, Spanish and Danish Codes); their requirements are considered to determine RES short-circuit current contributions.

The German codes [7][8][9] require WT to stay connected to the grid for voltages higher than 0.45 p.u. during the first $150 \mathrm{~ms}$ after the fault. For lower voltages, they allow short time disconnection with resynchronization within $2 \mathrm{~s}$; Wind Power Plants (WPP) are not allowed to disconnect from the grid, no matter the value of the residual voltage. WPPs are also required to increase the reactive current injected into the grid proportionally to the voltage drop. Further prescriptions are given on the reactive current for stability reasons.

The Spanish grid code [10] requires both LVRT and power injection requirements. About LVRT, the plant must remain connected for at least $150 \mathrm{~ms}$ in every condition of residual voltage for single line to ground or three-phase faults, while for line-to-line faults it can disconnect for voltage lower than 0.5 p.u. In the meantime, if the voltage varies more than $15 \%$ from the set point, a controller generates an instantaneous reactive current reference based on the mismatch with the voltage at the PCC. Once the fault is cleared, the controller is kept in service for at least 30 s after the voltage comes back to the normal range. Concerning active power injection, a 
limitation of the real current as a function of the pre-fault power and the residual voltage is required; it ensures a smooth restoration of the pre-fault power within $250 \mathrm{~ms}$ after the contingency clearance.

The Danish grid Code [11] requires a LVRT capability for three-phase faults; during single line or double line faults, the plant is only asked to withstand unsuccessful re-closures. No requirements on reactive current or power limitations during faults or clearance time are prescribed.

The prescriptions of the Italian Grid Code are detailed in Annex A17 [12] and A68 [13] for Wind and PV plants respectively, related in particular to plants connected at $\mathrm{HV}$ grids. In [12] and [13], updated in 2018, the LVRT characteristic is shown: WPP and PV plants must remain connected even with zero residual voltage and can not disconnect, in any case, before $0.2 \mathrm{~s}$. This requirement is different from what stated in previous versions, where disconnection was allowed for residual voltages lower than 0.2 p.u. (the latter prescription is adopted in the examples provided in Section V). There are not strict requirements on the injection to be kept during the fault, but the limitation of the real power has to be correlated to the voltage dip. The injections associated to phases not involved in the fault should be unaffected. The reactive power injection has to be restored within $2 \mathrm{~s}$. Noting that, the requirements for the voltage support is to be considered by the Italian TSO. For example, in [14], in addition to the specifications related to the LVRT, storage systems are required to support voltage during the fault by injecting an additional reactive current during both symmetrical and unsymmetrical faults, thus reducing the real power injection if it is necessary to keep the storage system within its limits. Such current must be proportional to the positive sequence voltage drop.

\section{F. A proposal for the computation of short-circuit current}

Usually, short-circuit calculations are carried out by considering only synchronous generators as sources. Traditional short-circuit calculation is based on the superposition principle. Although for IG directly connected to WT the IEEE model based on constant voltage behind a reactance is still appropriate, the behaviour of inverter interfaced RES like DFIG, FSC and PV plants is based on current injections related to the control strategy and, given their non-linear behaviour, do not allow using the superposition principle.

Hence, an iterative method has been implemented herein and tested in accordance with [15],[16] and [17]. The main idea is to iteratively evaluate the inverter injection as a function of pre-fault supplied power and voltage during the fault, by converting the inverter into an equivalent variable impedance.

As the details on converter controls are proprietary information and generally not known by TSOs, their behaviour should be based on the grid code. The German grid code is here used as an example, but other grid codes might be considered as well; accordingly, predetermined reactive current injections are required to be supplied within $20 \mathrm{~ms}$, making it reasonable to consider the inverter fed-in already under controller regulation in sub-transient state. The following assumptions are made:

a) if the voltage variation is lower than 0.1 p.u. (dead band), the pre-fault complex power is kept constant; b) the reactive injection follows the minimum requirement of the grid code: when the voltage at the PCC decreases more than $10 \%$, the quadrature current is raised to keep the voltage at the nominal value;

c) once incremental and pre-fault reactive current are added together, if the current is still lower than the maximum value (1.5 p.u.), the spare capability is used to supply the same real power as in pre-fault condition;

d) for DFIG, the same characteristic as for the FSC interfaced RES is considered until the voltage reduces below the crowbar threshold. Then, the DFIG is converted into an equivalent WRIG. This characteristic is typically adopted by manufacturers.

In the proposed procedure, a traditional short-circuit computation is first carried out neglecting inverters (i.e., modelling them as infinite impedances) and an initial appraisal for voltage profile is determined. Based on the voltage at converter terminals, new values of current injections are determined, considering the above-mentioned assumptions. An equivalent converter impedance is computed, and included into the impedance bus matrix, and an updated post fault voltage is computed. If that value is different compared to the previous iteration, the procedure proceeds to the next iteration; if not, the process is stopped and current that solution is accepted.

It is also necessary to consider the effect of inverter-based RES disconnection: after reaching convergence, the voltage of all RES under control is compared to the threshold for plant disconnection given by the grid code. Then, if some such generators go below the voltage disconnection limit, they are considered disconnected, and another iteration has to be carried out.

It is worth pointing out that as the RES penetration grows, the convergence might be difficult. For instance, that reason, for the Sicilian network which was adopted for testing (181 RES out of 261 generator), the number of iterations is limited to 15 when using a tolerance of $10^{-4}$ p.u. for on both active and reactive current. In case of convergence difficulty, a deceleration factor (more granular iterations) is sometimes necessary.

Figure 7 shows the errors on the computed values of shortcircuit power in every bus according to the proposed methodology (adopting the Eon Grid Code) and assuming infinite impedance of inverter-based generators. Errors are not negligible, reaching up to $12 \%$, thus justifying the need of the newly proposed procedure. For voltages a mean error of $3 \%$ and maximum errors of more than $50 \%$.

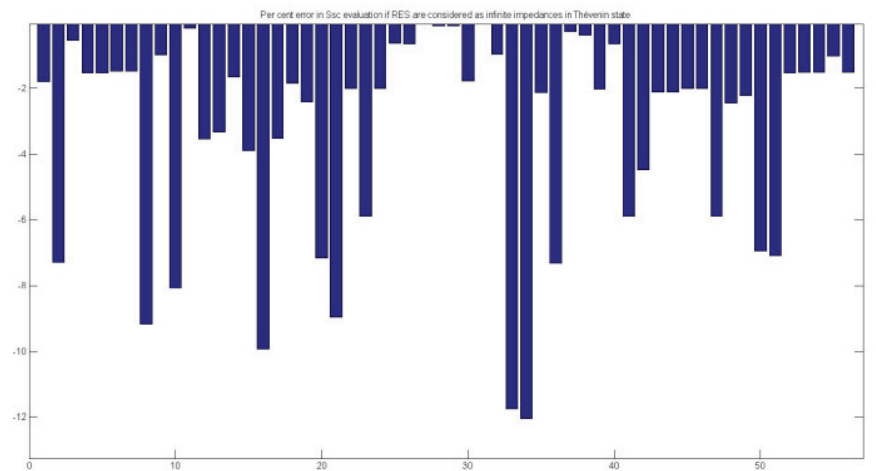

Figure 7 - Short-circuit power errors neglecting inverter-based RES contribution 


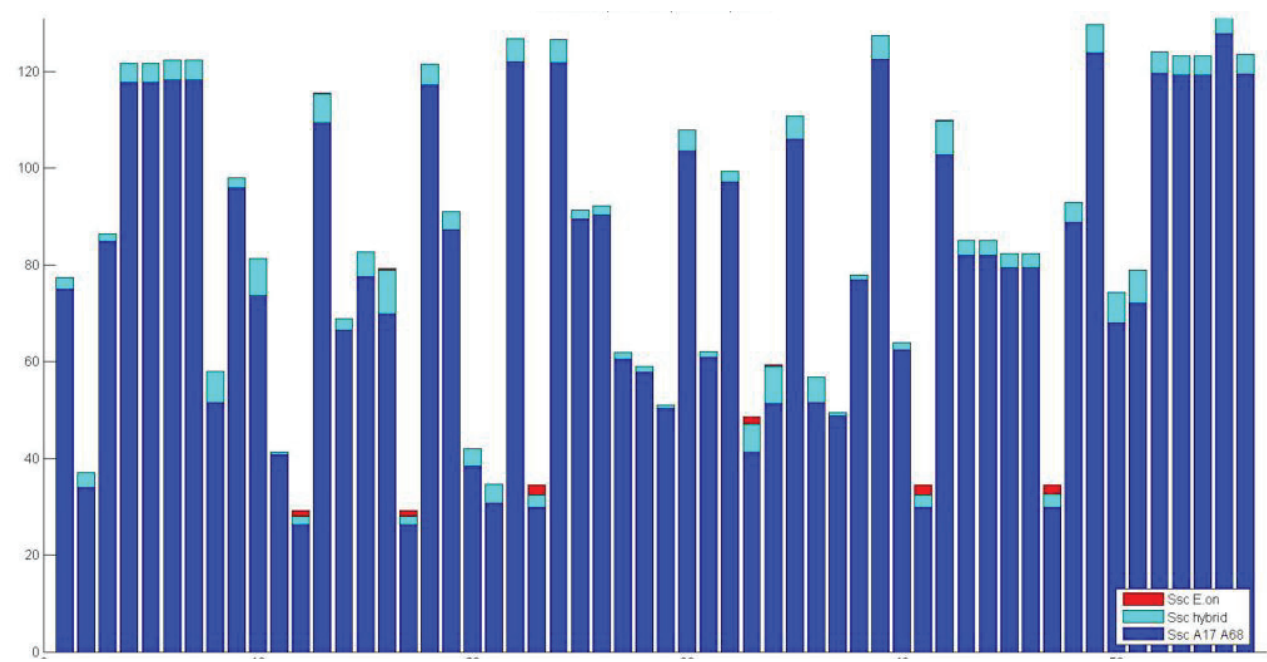

Figure 8 EHV bus short circuit power comparisons for the three control strategies applied to the base load scenario.
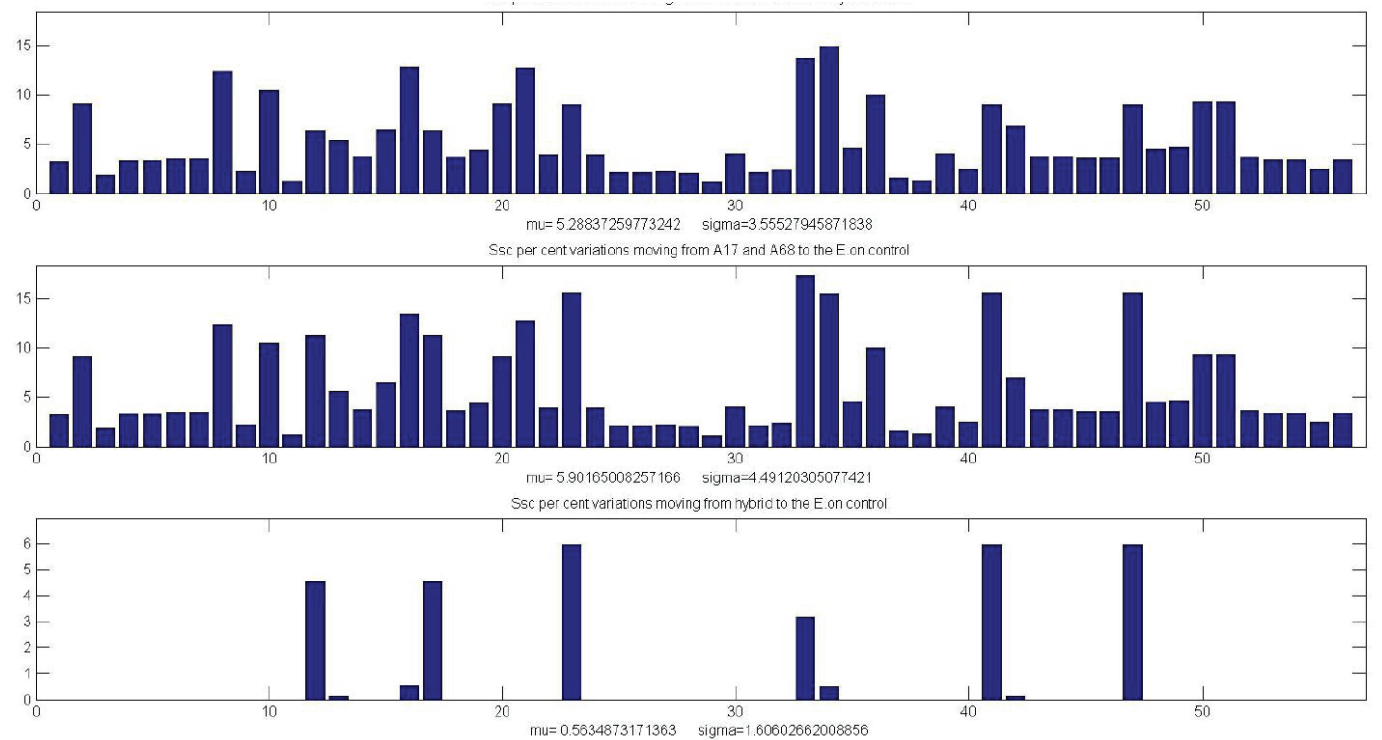

Figure 9 - 1st row: EHV bus short-circuit power per cent increase moving from the constant power injection to the hybrid control (from Case 1 to Case 2; 2nd row: EHV bus Ssc per cent increase moving from the constant power FRT to the E.on reactive current injection control (from Case 1 to Case 3); 3rd row: EHV bus Ssc per cent increase moving from Case 2 to Case 3.

\section{SCENARIOS CONSIDERED}

An equivalent model of the Sicilian transmission and subtransmission network is considered [18]. In the Base Case (BC), the total power generated is $4299.22 \mathrm{MW}$ and the total load is $4269.50 \mathrm{MW}$. The contribution of the PV and Wind power plants are respectively 33.56 MW and 365.08 MW. Moreover, further $662 \mathrm{MW}$ are provided by MV PV and Wind plants. The latter have not been considered for the evaluation of the proposed procedure.

\section{NUMERICAL RESULTS}

The proposed algorithm is used to compare the system response to different RES control strategies: from the simple constant power supply to the implementation of reactive current injections. Three cases are compared (Table I):

Case 1: HV RES control according to the Fault Ride Through (FRT) requirements of the former Terna grid code annexes (A17 and A68). Given that the procedure requires the specification of the supplied active and reactive current components, each WPP is assumed to inject the pre-fault complex power up to the maximum current limit of $1.5 I_{n}$. If the latter condition applies, the power is correspondingly reduced to a constant power factor. In case of disconnection, the plant is modelled as a null current source.

\begin{tabular}{|cccc|}
\hline & $\begin{array}{c}\text { Scenario 1: } \\
\text { Attachments A17 A68 }\end{array}$ & $\begin{array}{c}\text { Scenario 2: } \\
\text { Hybrid control }\end{array}$ & $\begin{array}{c}\text { Scenario 3: } \\
\text { E.On RCI }\end{array}$ \\
\hline HV RES control strategy & Constant power & $\mathrm{I}_{q}$ injection & $\mathrm{I}_{q}$ injection \\
\hline HV WPPs disconnection & $0.2 \mathrm{Vn}$ & $0.2 \mathrm{Vn}$ & $\sim$ \\
\hline Crowbar for HV DFIG & $0.4 \mathrm{Vn}$ & $0.4 \mathrm{Vn}$ & $\sim$ \\
\hline MV RES control strategy & Constant power & Constant power & Constant power \\
\hline MV WPPs disconnection & $\sim$ & $\sim$ & $\sim$ \\
\hline Crowbar for MV DFIG & $\sim$ & $\sim$ & $\sim$ \\
\hline
\end{tabular}

Table I - Cases considered for the simulations.

Finally, being the most common WT installed in the Sicilian network DFIG type, a crowbar protection is considered for residual terminal voltages lower than 0.4 p.u., converting the WT into an equivalent induction generator;

Case 2: This case is similar to Case 1, but all HV regulating inverter-based RES generators are assumed to control the reactive current injections $\mathrm{I}_{\mathrm{q}}$ accordingly to the E.On minimum requirements:

$I_{q}=I_{q 0}+\Delta I_{q}$ 
$\Delta I_{q}=2 \Delta V$

$\Delta V=V_{\text {pre-fault }}-V_{\text {short-circuit }}$

where $\mathrm{I}_{\mathrm{q}}$ is the reactive component of the current and $\mathrm{I}_{\mathrm{q} 0}$ is its pre-fault value

$\mathrm{I}_{\mathrm{q}}$ is limited to $1.5 I_{n}$ and in case some residual current capability is still present, it is used to transmit the pre-fault real power up to the maximum current.

Case 3: In the last case, neither disconnection nor crowbar protection is adopted.

In all cases, MV RES generators as well as their equivalents are considered as constant power injections with no disconnection allowed even for extra low voltages in accordance with the CEI 0-16 standard [17].

Case 1 describes the HV and MV RES behaviour in which only WPPs are allowed to disconnect from the grid in case of severe fault. Then, the assumption of constant power injection was considered as the best approximation of their physical behaviour, given that no power requirements have been required so far by the Italian transmission grid code. On the opposite side, Case 3 case aims at investigating the positive effects (from the voltage support point of view) if all HV RES followed reactive current requirements.

Finally, as Case 3 is not implemented in the actual grid due to the high costs of equipping DFIG WT generators with FRT capability (it is estimated that these requirements would increase the cost of DFIGs by 5\% [11]) the hybrid Case 2 represents a reasonable trade-off.

Moving to the simulation results, EHV bus short-circuit powers are compared in Figures 8 and 9.

Figure 8 depicts the p.u. short-circuit power (Ssc); it shows that moving from Case 1 to Case 3 the contribution to the short-circuit current increases, even at the transmission system level, showing a better and better voltage support. The same is better described in the first two layers of Figure 11, that shows the improvement when moving from the constant power control to the hybrid case (1st layer) and to the third control strategy (2nd layer) respectively. In both steps, the improvement is higher than $5 \%$, that justifying stricter requirement specifications. On the contrary, the analysis of the third row of Figure 9 makes the imposition of a complete FRT capability not justifiable due to the little additional improvement found.

\section{CONCLUSIONS}

Being the majority of the new RES installations inverter interfaced to the grid, it is necessary to analyse their effects on the system security and quality of the supply. In particular, the reduction of the short-circuit power is investigated in this paper and a steady-state procedure to compute the shortcircuit current in presence of a high penetration of RES is taken into account and tested, going beyond the usual practice of neglecting converters within the Thevénin state or, similarly, modelling them as extremely high impedances. The effects of different RES requirements from different Grid Codes (e.g. continuous operation even in case of significant voltage dip and support to the voltage recovery after a fault) have been investigated in terms of network strength. In particular, the iterative procedure developed is able to take into account the LVRT characteristic and the real and reactive power injections as required by the Grid Codes. Particularly interesting is the set of prescriptions on the reactive current injection, considered by the German and the Spanish Grid Codes, which also Terna is taking into account in its technical specification for future storage systems.

\section{REFERENCES}

[1] Attachment A8 of Terna's Grid Code, Correnti di corto circuito e tempo di eliminazione dei guasti negli impianti delle reti a tensione uguale o superiore a $120 \mathrm{kV}$ (in Italian) ). Available at www.terna.it

[2] Terna Grid Code. Available at https://www.terna.it/itit/sistemaelettrico/codicedirete.aspx

[3] IEEE PES Technical Report PES TR-22, Contribution to bulk power system control and stability by Distributed Energy Resources connected at distribution network, IEEE 2013,

[4] R. A. Walling, E. Gursoy, B. English, Current Contributions from Type 3 and Type 4 Wind Turbine Generators During Faults, IEEE/PES Transmission and Distribution Conference and Exposition (T\&D), 2012.

[5] G. Xiang, W. Gang, W. Zhengrong, L. Haifeng, Fault Current Contributions of Doubly Fed Induction Generator Wind Turbines under Different Control Strategies, International conference on Advanced Power Sysytem Automation and Protection (APAP) 2011.

[6] Thomas Ackermann, Wind power in power systems, 2nd Edition, John Wiley \& Sons, 2012.

[7] E.on Netz GmbH, Bayreuth, Grid Code High and Extra High Voltage, 1st April 2008. Available at: http://www.eon-netz.com.

[8] Ordinance on System Services by Wind Energy Plants (System Service Ordinance - SDLWindV) - Draft, 2009. Available at: http://www.erneuerbare-energien.de

[9] Transmission Code 2007. Networksand System Rules of the German Transmission System operators, VDN-e.v. beim VDEW, August 2007. Available at http://www.vde.com/de.

[10] M. Altın, O. Goksu, R. Teodorescu, P. Rodriguez, B. Bak-Jensen, L. Helle, Overview of Recent Grid Codes for Wind Power Integration, International conference on Optimization of Electrical and Electronic Equipment (OPTIM), 2010.

[11] Grid connection of wind turbines to networks with voltages above 100 $\mathrm{kV}$, Regulation TF 3.2.5, Eltra and Elkraft System. Available at http://www.wt-certification.dk.

[12] Attachment A17 of Terna Grid Code, Condizioni generali di connessione alle reti AT. Sistemi di protezione, regolazione e controllo (in Italian), 2018. Available at www.terna.it.

[13] Attachment A68 of Terna Grid Code, Centrali fotovoltaiche. Condizioni generali di connessione alla rete AT. (in Italian), 2018. Available at www.terna.it.

[14] Progettazione e realizzazione di un sistema di accumulo elettrochimico da connettere alla rete di trasmissione nazionale $(132-150 \mathrm{kV})$. Terna technical requirements (in Italian), 2014.

[15] O. Goksu, R. Teodorescu, B. Bak-Jensen, F. Iov, P. C. Kjar, An Iterative Approach for Symmetrical and Asymmetrical Short-Circuit Calculations with Converter-Based Connected Renewable Energy Sources. Application to Wind Power, IEEE Power and Energy Society General Meeting, 2012.

[16] M. Valentini, V. Akhmatov, F. Iov, J. Thisted, Fault Current Contribution from VSC-based Wind Turbines to the Grid, The Second International Symposium on Electrical and Electronics Engineering ISEEE-2008, Galati, Romania.

[17] CEI 0-16, Reference technical rules for the connection of active and passive consumers to the $\mathrm{HV}$ and $\mathrm{MV}$ electrical networks of distribution Company, 3rd edition, December 2012. Available at: http://www.autorita.energia.it.

[18] D.Polinelli, Preliminary study on the voltage regulation in presence of high RES penetration, MSc Thesis, Politecnico di Milano, 2012-1013. 